\title{
Complications of Coinfection with Babesia and Lyme Disease After Splenectomy
}

\author{
Ya'aqov Abrams, $M D$
}

The patient is a 58-year-old man who had a trauma-related splenectomy 25 years ago. In August 2002 he presented to the office with several days of fluctuating fevers of $102^{\circ} \mathrm{F}$ or higher, rash, malaise, chills, and sweats. He spent a week at Cape Cod before a trip abroad to Brazil, from which he had just returned. His symptoms developed after the first week in Rio de Janeiro, Brazil. He recalled having no mosquito or other insect bites while in Brazil or Cape Cod. His temperature was $100.5^{\circ} \mathrm{F}$ and he had an eyrthema multiforme-like macular rash on his back and abdomen. An infectious disease specialist was consulted. Laboratory studies were notable for bandemia of $11 \%$, mildly elevated liver injury tests, normal bilirubin, negative babesiosis, ehrlichia, Rocky Mountain Spotted Fever and typhus immunoglobulin $\operatorname{IgG}$ and $\operatorname{IgM}$ antibody titers, negative hepatitis A and hepatitis B titers, and a negative thick smear for intra-erythrocytic parasites. He was prescribed doxycycline but did not improve. Twelve days after presentation he noted myalgias with associated difficulty walking and was seen in the emergency department. Intraerythrocytic parasites were noted on a thick blood smear; malaria was diagnosed and the patient was prescribed oral chloroquine.

One day later the diagnosis was changed to babesiosis and treatment was changed to oral clindamycin and quinine. Several days later the patient was hospitalized with dark-colored urine and Babesia parasitemia of $4 \%$. He initially received intravenous quinine and clindamycin, but he did not

This article was externally peer reviewed.

Submitted 22 October 2006; revised 27 July 2007; accepted 7 August 2007.

From the Department of Family and Community Medicine, University of Pittsburgh, PA.

Funding: none.

Conflict of interest: none declared.

Corresponding author: Ya'aqov M. Abrams, Department of Family and Community Medicine, University of Pittsburgh, 5608 Wilkins Ave, Pittsburgh, PA 15217 (e-mail: abramsym@upmc.edu). respond and treatment was changed to atovaquone, azithromycin, and a 2-week course of doxycycline. He developed adult respiratory distress syndrome, presumably from a Babesia parasitemia now up to $16 \%$; this necessitated an exchange transfusion. The myalgias and weakness worsened and primary demyelinating polyneuropathy was diagnosed on electromyography. A magnetic resonance image of the brain was normal but serum IgM and Western Blotting were positive for Lyme disease. Despite a 1-month course of ceftriaxone, neuropathic symptoms did not improve until he received a 5-day course of intravenous immune globulin (IVIG). Five years later, the patient still has mild residual lower extremity sensory neuropathy but is otherwise well. He did not recall previously receiving vaccinations for encapsulated bacteria and was given meningitis, Haemophilus, and pneumococcal vaccines.

\section{Discussion}

At the initial presentation the patient's visit to Cape Cod was not mentioned and the differential diagnosis focused on his history of travel to Brazil. However, because July and August are winter months in South America, the transmission of mosquito-borne diseases in Brazil was, in hindsight, unlikely. Knowing this might have directed the diagnosis back to infectious sources from the United States and may have changed empiric therapy to treatment of babesiosis (known to be a potentially fatal infection in a splenectomized patient). Coinfection with Babesia and Lyme disease is common. ${ }^{1}$ Some suggest that ticks may host multiple infective agents and that this may increase the number of diseases a patient may present with after a tick bite. ${ }^{2,3}$ This patient presented with symptoms and signs of 2 diseases. His fever and malaise were most likely caused by babesiosis; his rash is best explained by stage 2 Lyme disease (note that rash is not a feature of babesiosis or malaria). Given his 


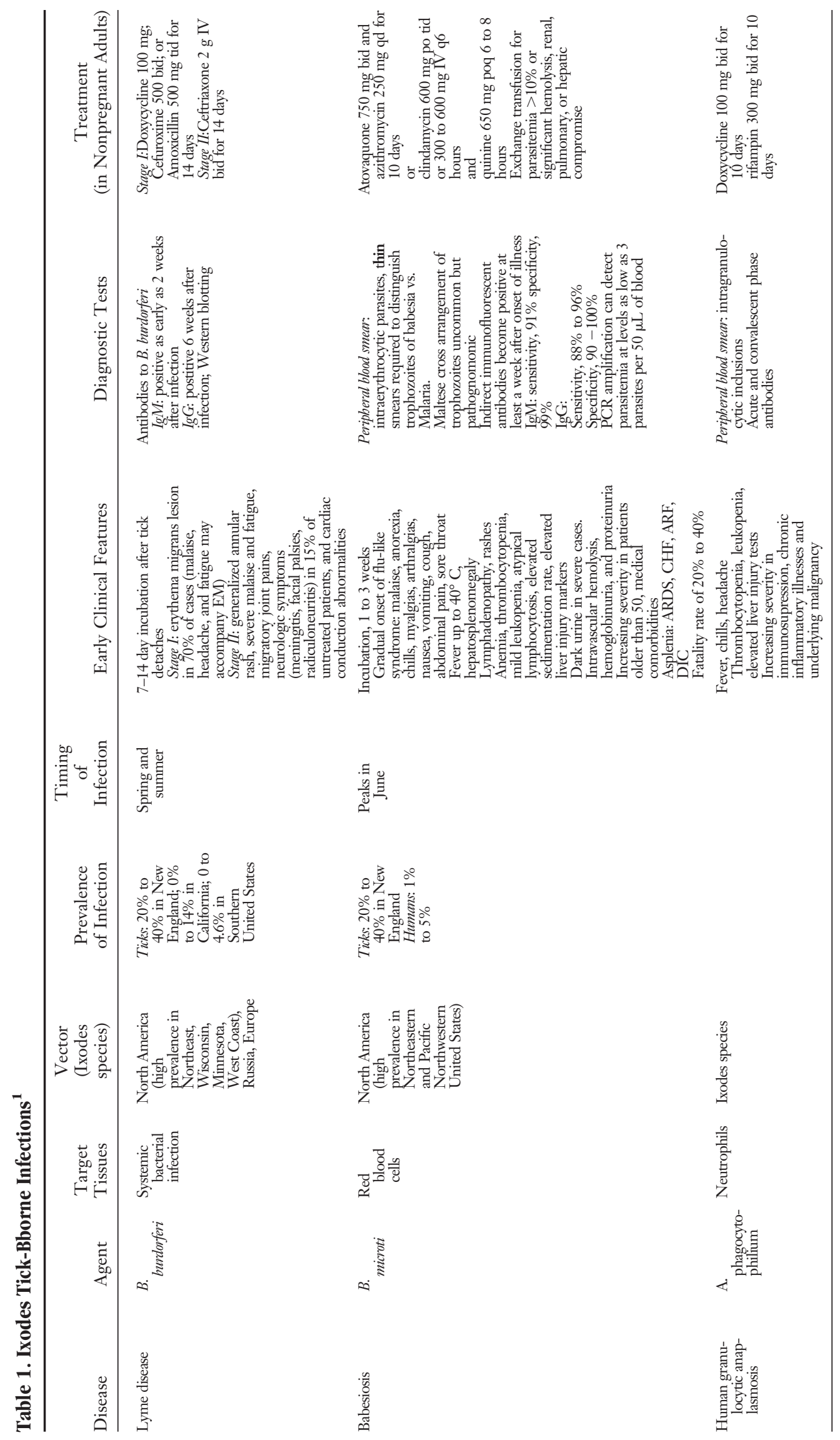


medical and travel histories, a peripheral blood smear showing intra-erythrocytic parasites was most suggestive of babesiosis. Peripheral blood smear is the definitive method of diagnosis of babesiosis, but thin blood smears are usually required to distinguish between the trophozoites of Babesia and Malaria. Polymerase chain reaction amplification can detect parasitemia at levels as low as 3 parasites per $50 \mu \mathrm{L}$ of blood, which can provide a diagnosis within 24 hours. IgM and IgG indirect immunofluorescent antibody tests have adequate sensitivity and specificity for diagnostic purposes (IgM, 91\% to $99 \%$; $\operatorname{IgG}, 88 \%$ to $96 \%$ and $90 \%$ to $100 \%$, respectively). However, antibodies do not develop for at least a week after the onset of illness, rendering serology less useful in the early diagnosis of acute infection. Treatment failures are common in babesiosis and Lyme disease, thus the need to change antibiotics during treatment is an anticipated complication. $^{4}$

Although neuroborreliosis may respond to IVIG, it is not known by what mechanism patients respond. IVIG has an off-label indication for therapy of neuroborreloisis. In the last published report on this topic, Crisp and Ashby ${ }^{5}$ proposed that the immune-modulating properties of IVIG might ameliorate Lyme disease by any of the following mechanisms: inhibition of cytokines, competition with autoantibodies, inhibition of complement deposition, interference with $\mathrm{Fc}$ receptor binding on macrophages or B-cells, or interference with T-cell recognition of antigens. ${ }^{5}$

This case highlights the importance of a precise and detailed travel history in the febrile traveler and, in particular, one who has a history of splenectomy. Patients with immunocompromised systems are at greater risk for a more prolonged and severe courses of illness, especially with multiple infectious etiologies, illustrated here with Lyme disease and babesia. ${ }^{6}$ In these patients, reasoning to the single most likely cause of illness may not be the best approach to diagnosis and empiric treatment. Familiarity with tick-borne diseases is important and may become more so as the habitats of humans and ticks increasingly intersect. ${ }^{7}$ Knowledge of which supporting and confirmatory laboratory tests to order is useful in expediting diagnosis and treatment. Finally, when treating any disease it is valuable to know which treatments are based on reliable research, such as the antibiotic choices for babesiosis and Lyme disease, and those which are anecdotal, such as IVIG treatment of neuroborreliosis.

We would like to thank Drs. William Markle and Emanuel Vergis for their editorial comments, and the patient, for allowing us to present his case.

\section{References}

1. Swanson SJ, Neitzel D, Reed KD, Belongia EA. Coinfections acquired from ixodes ticks Clin Microbiol Rev 2006;19:708-27.

2. Alekseev AN, Semenov AV, Dubinina HV. Evidence of Babesia microti infection in multi-infected Ixodes persulcatus ticks in Russia. Exp Appl Acarol 2003;29: $345-53$.

3. Adelson ME, Rao RV, Tilton RC, et al. Prevalence of Borrelia burgdorferi, Bartonella spp., Babesia microti, and Anaplasma phagocytophila in Ixodes scapularis ticks collected in Northern New Jersey. J Clin Microbiol 2004;42:2799-801.

4. Wormser GP, Dattwyler RJ, Shapiro ED, et al. The clinical assessment, treatment, and prevention of lyme disease, human granulocytic anaplasmosis and babesiosis; clinical practice guidelines by the Infectious Diseases Society of America. Clin Infect Dis 2006;43:1089-134.

5. Crisp D, Ashby P. Lyme radiculoneuritis treated with intravenous immunoglobulin. Neurology 1996; 46:1174-5.

6. Krause PJ, Telford SR $3^{\text {rd }}$, Spielman A, et al. Concurrent Lyme disease and babesiosis. Evidence for increased severity and duration of illness. JAMA 1996;275:1657-60.

7. Comer JA, Paddock CD, Childs JE. Urban zoonoses caused by Bartonella, Coxiella, Ebrlichia, and Rickettsia species. Vector Borne Zoonotic Dis 2001;1:91-118. 